\title{
CORPORALIDADES Y SEXUALIDADES: DEL VÍNCULO A LAS FRACTURAS SOCIALES
}

\author{
José Sánchez-Parga
}

\section{Preámbulo hermafrodita}

Los días 26 y 27 de noviembre, con motivo de la Semana de Psicología Social, estudiantes de la carrera pintaron un gran mural, la figura en cuestión era monstruosa, una suerte de hermafrodita: un cuerpo humano con dos cabezas, una femenina y otra masculina, $y$ una vagina junto a un pene. A mi pregunta intrigada respondieron que simbolizaba la unión del hombre y la mujer. La respuesta sorprende más que la misma figura, porque no hay unión posible, allí donde ya hay unidad.

De hecho es imposible que el pene del hombre pueda penetrar la vagina de la mujer, cuando ambos sexos se encuentran ya unidos, uno junto al otro, en un mismo cuerpo. Más aún, la unión del hombre y la mujer no es únicamente la de sus sexos sino también la de sus cuerpos y la de sus personales subjetividades, todavía más imposible cuando sólo hay un cuerpo. De otro lado, un sím- bolo nunca tiene una representación real, sino al contrario: es la realidad la que puede ser simbólicamente representada por medio de una imagen para producir una significación. Finalmente, la figura hermafrodita — pintada por los estudiantes - es una supresión de la alteridad, del "otro" sujeto individual y diferente, ya que la diferencia personal entre el hombre y la mujer supone la existencia de dos cuerpos diferentes, puesto que el cuerpo es el principio de individuación de la persona, y por consiguiente de su subjetividad. Y si la diferencia es "ser-para-un-otro" (según Hegel), la diferencia corporal significa que un cuerpo "es-paraotro" cuerpo; de la misma manera que sólo la absoluta diferencia (y no sólo corporal como se verá más adelante) entre el hombre y la mujer los define como "uno-para-otro".

En conclusión, sin un "otro" diferente no hay posible relación personal ni vínculo social posible. No es suprimiendo la diferencia entre los

\footnotetext{
* Profesor de la Universidad Politécnica Salesiana.
} 
sexos, que se puede afirmar la igualdad entre ellos, sino todo lo contrario, ya que la igualdad (que es una relación) presupone la diferencia (que también es una relación), tanto como ésta requiere aquélla. Es desde la real diferencia entre los sexos que es posible construir una igualdad entre ellos, un mutuo "re-conocimiento" e identificación.

Al suprimirse las relaciones y vínculos, tanto sociales como sexuales, resulta imposible toda representación/función simbólica, puesto que el símbolo (de acuerdo a su misma etimología griega, sym-ballein) no es más que producción y producto del vínculo social. Esto explica que los jóvenes estudiantes representen el hombre y la mujer confundidos en la materialidad de un solo cuerpo, y que dicha fusión corporal sea un imaginario tan fantasmal como monstruoso. $\mathrm{Al}$ estar unidos por un mismo cuerpo, hombre y mujer, lejos de lograr una unión imposible entre ellos, sólo pueden ser uno mismo, plenamente hombre y plenamente mujer sacrificando o eliminando al "otro". Porque el ideal de la masculinidad y de la feminidad no puede ser su total fusionalidad y total indeterminación o indiferenciación corporales y sexuales, sino todo lo contrario: es en la más absoluta diferencia con respecto a la mujer que se cons- truye la masculinidad y en la más absoluta diferencia con respecto al hombre que se construye la feminidad.

La anécdota que ha servido de punto de partida a estos planteamientos introductorios resulta muy ilustrativa de los profundos cambios operados durante las últimas décadas en la relación con el cuerpo y su representación tanto como respecto a la sexualidad, las maneras de pensarla y vivirla. Más aún, hoy son tantas las formas que adopta el cuerpo humano como diversas las corporalidades y sexualidades, y así también los discursos sobre ellas. Esto ha hecho que en torno a la corporalidad y sexualidad humanas las confusiones sean muchas y los enfrentamientos que provocan tan tenaces.

En el origen de estas mutaciones y de las diversas fenomenologías relativas al cuerpo y la sexualidad el principal factor explicativo es un neoindividualismo egoísta y narcisista, hedonista y posesivo. Este nuevo individualismo, producto de la sociedad de mercado, es el que ha transformado las representaciones y relaciones del hombre moderno con el cuerpo y la sexualidad, convirtiendo lo que siempre fue un vínculo y producto de vínculos humanos en instrumentos de confrontaciones y campos de lucha de unos contra "otros". 
Sin embargo, esta radical mutación de las corporalidades y sexualidades es un exponente y expresión —quizás uno de los más significativos- de la profunda mutación antropológica que tiene lugar en la moderna sociedad de mercado. Si, según Marx, el capital no sólo produce mercancías para el hombre, sino también un hombre - hombrespara la mercancía, habría que suponer que las nuevas corporalidades y sexualidades son, sobre todo, producto de intercambios mercantiles, de ofertas y demandas, de circulación, marketing y consumo.

En este sentido, la moderna sociedad de mercado no se limita a producir nuevas relaciones con el cuerpo y la sexualidad y nuevas relaciones corporales y sexuales sino que, además, desarrolla sus propios medios de producción con sus correspondientes técnicas y tecnologías para producir las nuevas corporalidades y sexualidades. Existe una colosal industria de la cosmética, de las más rentables económicamente, todo un repertorio de "productos de belleza" que va desde los perfumes más sofisticados hasta los energizantes o la bio-farmacéutica para los "rejuvenecimientos" de todas las partes del cuerpo, pasando por las más variadas cirugías estéticas; entre otras formas adoptadas por el culto al cuerpo se multiplican las dietas, los fitness y los gimnasios, los bodybuilding, proliferan los saunas y spa; toda una panoplia de artes orientales y más o menos marciales, para el control del propio cuerpo (y del "otro") se exportan, difunden y venden por todos los medios sociales. El culto al cuerpo hoy está cada vez más asociado con el deporte, y el imperativo de "estar en forma" constituye el sinónimo de mantenerse joven, atractivo y dinámico. El look y la apariencia, la "presentación", se vuelven un dominante tanto como una obsesión.

El extraordinario desarrollo que la moda, la industria del vestuario ha alcanzado en la sociedad moderna se explica en gran medida por la relación que ésta tiene con el adorno y la exposición del cuerpo - las marcas corporales-, y por poner de relieve las anatomías de los sexos, y, de manera más reciente, los vestidos se han convertido en una manera estilizada de desnudar los cuerpos, insinuándolos más que vistiéndolos. Así es como la ropa se ha convertido en un referente de identidad, un significante del "gusto" y la personalidad, que uniformiza a la vez que individualiza; ya que paradójicamente "estar a la moda" es vestir como todos pero con la ilusión de vestirse cada uno a su manera. El 
mismo modelo de la "alta costura" se reproduce en la ropa en serie o pret-a-porter.

El cuerpo humano pasa hoy por todas las manipulaciones y tecnologías: desde mantenerlo "en coma" o estado vegetativo durante años hasta usar sus órganos para traficarlos; pasando por los implantes o transplantes más audaces de la cirugía médica y por las fantasías de los percing y tatuajes. No ajeno a esta moderna manipulación del cuerpo humano es el desarrollo de las más crueles técnicas de tortura. En resumen, el cuerpo humano se ha vuelto tanto el lugar de todos los placeres y goces como el de todos los horrores y sufrimientos; y quizá más de enfrentamientos que de encuentros.

En conclusión, la figura del hermafrodita, de la fantasmal supresión de la división sexual, supone la supresión de la alteridad, del "otro"; es decir, supresión del deseo, supresión de la carencia fundamental; en definitiva, supresión de la sexualidad, ya que ésta no es más que la encrucijada de tres realidades: el otro, el deseo y la carencia fundamental, que definen la esencia del ser humano.

\section{Dilema entre ser o tener un cuerpo}

El cuerpo humano es una invención relativamente reciente. En las sociedades primitivas el cuerpo humano en cuanto representación individual de las personas no existía, hasta el punto de que en sus lenguas ni siquiera había la palabra cuerpo. En la lengua kichwa de las sociedades andinas la referencia al cuerpo humano se designa con la palabra aycha (carne); que es curiosamente lo mismo que ocurre en el antiguo hebreo, en el cual en lugar del término cuerpo se usa la palabra bashar, que significa carne, en el sentido de cuerpo humano y de ser humano ${ }^{1}$. En estas sociedades comunales o corporativas, investidas por una fuerte personalidad colectiva, los individuos se consideraban miembros del cuerpo social, orgánicamente vinculados entre sí.

"La formulación de la palabra cuerpo en cuanto fragmento en cierto modo autónomo del hombre, del que lleva su rostro, presupone una distinción ajena a numerosas comu-

1 En el prólogo del IV Evangelio la frase: "el Verbo se hizo carne", significa cuerpo y ser humano, persona humana. 
nidades humanas"2. Una cosa es que el indígena posea una representación exterior del cuerpo, conozca su superficie y sea capaz de nombrar muchas de sus partes, y otra cosa muy distinta consiste en saber que su cuerpo constituye una unidad tan íntima como inseparable de sí misma; entre ambas experiencias y representaciones del cuerpo hay un abismo: "es el mismo abismo que separa el primitivo del hombre moderno" 3 . En las sociedades modernas, societales, el cuerpo es factor de individuación, mientras que "en las sociedades primitivas el cuerpo no se distingue de la persona"4.

Para el hombre andino, como para los canaca melanesios, estudiados por Leenhardt, el cuerpo no es objetivable, no representa una realidad exterior que pueda ser aislada, singularizada y diferenciada de sí misma, algo que la persona tiene; $y$ en tal sentido el cuerpo lejos de ser el límite que diferencia y separa una persona de otra, convirtiéndose en el principio material y concreto de la individualización, posee una dimensión colectiva que todos comparten y que los hace miembros del mismo cuerpo social. Según esto, para las sociedades comunales, o primitivas, el cuerpo no es objetivable, algo exterior que pueda ser singularmente aislado, diferente de la persona misma y que ésta posee. En las sociedades comunales la identidad personal no se delimita por el cuerpo, ya que "éste no separa del grupo sino que por el contrario lo incluye en él" (Le Breton: 1990: 25).

En la tradicional sociedad comunal andina "el cuerpo no existe en cuanto elemento de individuación, puesto que el individuo mismo no se distingue del grupo, todo lo más es una singularidad en la armonía diferencial del grupo" (Le Breton, 1990: 23). A diferencia del hombre andino que es un cuerpo (carne, aycha) en la medida en que pertenece a un cuerpo, que comparte con todas las demás

2 David Le Breton, Anthropologie du corps et modernité, PUF, Paris, 1990:22. Y el autor precisa que "en las sociedades tradicionales de carácter holista, comunitario, donde el individuo es indiscernible, el cuerpo no es más objeto de una escisión, y el hombre está incorporado al cosmos, la naturaleza y la comunidad" (ibíd.).

3 Según Maurice Leenhardt (Do Kamo. La personne et le mythe dans le monde mélanésien, Gallimard, Paris, 1971:54) "el primitivo es el hombre que no ha aprendido el vínculo que lo une a su cuerpo y que desde entonces ha quedado incapaz de singularizarlo" (p. 70).

4 Cfr. Emile Durkheim, Formes élémentaires de la vie religieuse, PUF, Paris, 1968:386s. 
personas de su grupo, y por consiguiente como el canaca melanesio "ignora su cuerpo" (o.c.: 152), el individuo moderno de la sociedad societal tiene un cuerpo, con el que puede mantener una particular relación. Por ello cabe hablar no sólo de una "personalidad colectiva" en la sociedad comunal sino también de una "persona participativa" (Leenhardt), ya que todos los individuos comparten un mismo cuerpo social, del que viven y se interpretan como miembros 5 .

Ya en las sociedades antiguas (Grecia y Roma) el cuerpo humano adquiere un valor individual, considerado soporte de la persona y de sus derechos ciudadanos, y por consiguiente exento de todo maltrato o castigo físico, puesto que la integridad física del cuerpo se identificaba con la integridad personal del individuo. En el posterior transcurso de la Historia y de las sucesivas transformaciones sociales, el proceso de creciente individualización de las personas comportará un cambio en la representación y su relación con el cuerpo: la sociedad societal, sin abolir lo común y colectivo, lo supedita cada vez más a lo individual, y así la corporalidad individual adquiere mayor presencia que la corporalidad social, y los individuos dejan de representarse y comportarse como miembros de un grupo u organismo social para relacionarse entre ellos y con el mismo grupo desde su propia individualidad personal, haciendo de sus propios cuerpos la mediación simbólica tanto de sus reconocimientos mutuos como de sus relaciones y vínculos más personales.

La identidad personal nunca se había encontrado limitada por el cuerpo, que, lejos de separar al individuo del grupo y de los otros individuos, era el medio y símbolo de su inclusión en el grupo y de su vinculación con los "otros"; por el contrario, hoy el cuerpo aparece como frontera entre el individuo y el grupo al que ya no pertenece, y entre el individuo y los otros individuos, uno $\mathrm{y}$ otros atrincherados en sus propios cuerpos.

Sólo a condición de dejar de pertenecer a un cuerpo social llega el individuo a definirse por ser y tener un cuerpo, pero sin ser ni tener totalmente su cuerpo. No es lo mismo tener un cuerpo como si fuera una propiedad cualquiera o como perte-

5 En canaca la palabra karo significa un elemento sustentador del hombre (kamo), el cual sería común a todas las personas del grupo; lo que todos comparten en cuanto miembros (Leenhardt). 
necer a la propia infancia o lugar de origen. La diferencia entre ambas posesiones es fundamental, ya que en un caso se puede hacer del cuerpo lo que uno quiere, $y$ en el segundo caso uno tiene deberes y obligaciones, responsabilidades sociales respecto al propio cuerpo. Y de otro lado, sostener que uno es su cuerpo no puede significar que la persona se limita a ser un cuerpo; nunca existimos fuera del cuerpo como tampoco existimos dentro de él. En cualquiera de los casos hay que aceptar y reconocer siempre, con Lacan, que "el cuerpo es el lugar del Otro".

Porque sólo en parte es y sólo en parte posee un cuerpo puede el individuo relacionarse personalmente con otros individuos y vincularse en cuanto sujeto con otros sujetos, haciendo del cuerpo una mediación de dichas relaciones. Por el contrario, si el cuerpo fuera total y exclusivamente propiedad del individuo o por el contrario éste se identificara total o subjetivamente con su propio cuerpo, aquél nunca podría ser vínculo social entre las personas. Un cuerpo totalmente poseído y propiedad del individuo sería un cuerpo objeto, desubjetivado, y por ello mismo sólo instrumento de relación con las personas, pero no vínculo intersubjetivo entre ellas. $\mathrm{Si}$, por el contrario, el individuo se identifica completamente con su cuerpo, se hace su cuerpo, tal subjetivación del cuerpo lo convertiría en el "alter ego" del individuo, el cual no sería capaz de una relación intersubjetiva con otras personas.

Cuando tener un cuerpo supone una tal objetivación del cuerpo propio y el de los otros, se convierte en un impedimento para a través de él poder establecer relaciones intersubjetivas con los "otros" individuos; los cuerpos así desubjetivados se hacen instrumentos del propio placer a costa del "otro" y de lucha entre las personas. Por otro lado, cuando ser un cuerpo comporta una tal subjetivación del propio cuerpo, que se vuelve un "alter ego", las relaciones con dicho cuerpo así subjetivado impiden la relación personal con "otros" sujetos.

Objetivado o subjetivado hoy "el cuerpo se ha vuelto y se ha impuesto progresivamente como una mercancía atrapada en los circuitos del consumo", y este interés mercantil del cuerpo se ha extendido a todas sus prácticas y sus usos ${ }^{6}$. El cuerpo siem- 
pre había sido metáfora susceptible de declinarse en una diversidad de símbolos, según las sociedades y culturas, hasta que en el mundo actual, investido por la plusvalía de la oferta y la demanda, tiende a concretarse en la forma de mercancía; y de lugar simbólico de intercambios, vínculos sociales y personales, el cuerpo se convierte en el lugar privilegiado de todas las luchas (de clases, de sexos, generacionales, religiosas y raciales) y de todas las violencias.

En todas las formas que adopta, el nuevo modelo de individualismo moderno se encuentra investido por el cuerpo, los individualismos egoístas, hedonistas, narcisistas, consumistas y competitivos son todos discursos e ideologías corporales, que se aprovechan del propio cuerpo a costa del de los "otros".

La desexualización del cuerpo humano, o la feminización del cuerpo del hombre y la masculinización del cuerpo de la mujer, al pretender eliminar la diferencia suprime lo que ésta significa "ser-para-un-otro" (según Hegel); y siendo la diferencia entre los sexos fundamental para el ser humano, su supresión es un exponente de la eliminación de todas las otras diferencias sociales $y$, por consiguiente, de ese "ser-para-losotros" constitutivo de lo social y principio de toda ética, según el mismo Hegel.

\section{Construcción y descons- trucción de la identidad sexual}

La sexualidad humana es esencialmente dual ya desde su fundamentación genética, y las tres fases o niveles de su desarrollo orgánico, sociocultural e individual marcan de manera distinta el carácter de su alteridad o relación al otro sexo. Mientras que la dualidad o alteridad somática está dada por la diferente genitalidad, y la socio-cultural por todo lo que diferencia lo masculino y femenino en sus comportamientos y estilos de vida, la dualidad y alteridad entre cada hombre y mujer se define por las relaciones que se establecen entre ellos. En otras palabras, mientras que la alteridad y diferencia orgánicas entre hombres y mujeres tienen una fijación somática, y las socio-culturales son históricamente cambiantes, las individuales dependen de cada persona.

La diferencia somática masculina y femenina, con la que nacen todos los seres humanos, será elaborada culturalmente por cada sociedad de acuerdo a las representaciones y prácticas propias de cada una de 
ellas, la que tenderá a definir y diferenciar las diferencias entre hombres y mujeres a su interior. Es cada sociedad y cultura las que definen las conductas y comportamientos, estilos y valores, maneras de ser, de pensar y de sentir propios de hombres y mujeres, y todo lo que los diferencia entre ellos, y que puede cambiar de una sociedad a otra. Tal diferenciación sociocultural entre los dos sexos se marcará cada vez más a lo largo de la infancia y sobre todo en la adolescencia, con la finalidad de establecer las condiciones para la tercera y definitiva fase de construcción de la sexualidad masculina y femenina, la cual se elabora y desarrolla a partir de la relación e intercambios entre hombre y mujer. Finalmente, es respecto al varón que la mujer define su feminidad y es respecto a la mujer que el hombre termina definiendo su masculinidad ${ }^{7}$.

Esta fase terminal en la construcción de la sexualidad masculina $y$ femenina, y que constituye un nivel meta-orgánico y meta-sociocultural, tiene por objeto la determinación más fundamental de la condición humana, pero también la que trans- ciende tanto lo natural y corporal como lo social y cultural: el deseo. Son las dos diferentes formas que adopta el deseo del "otro" lo que termina por definir lo que es un hombre y una mujer: el hombre es deseo de la mujer, y la mujer es deseo de ser deseada por el hombre. De esta manera, la construcción de las identidades sexuales, aun teniendo una base orgánica y una fase o forma sociocultural, transciende dichas determinaciones somáticas y culturales para completarse y terminar definiéndose o precisándose a partir de la relación entre ambos sexos y del reconocimiento de su alteridad; lo que a su vez comporta una mutua identificación que condiciona el vínculo entre ellos.

Es importante notar que el deseo humano es dual no sólo en el ámbito de la sexualidad sino también del poder, como muy bien lo pensó Maquiavelo al fundarlo en "el deseo de dominar y de no ser dominado"; ambos momentos o fases del mismo deseo tanto se complementan como se contradicen. Así como el deseo de dominar es inherente al deseo de no ser dominado (dominar para no ser

7 Según Martine Lerude hay un primer nivel subjetivo en la construcción de la sexualidad que recorre cada niño y adolescente, un nivel objetivo o social y un tercer nivel de la relación con el otro sexo La feminidad: ¿cómo se construye?', en Ecuador Debate, n. 59, agosto, 2003. 
dominado), de igual manera el deseo masculino de la mujer no sería posible sin el deseo femenino de ser deseada, como tampoco este deseo de la mujer sería posible sin el deseo del hombre. Es a este nivel, tan fundamental del psiquismo y de la misma naturaleza humana, que se instituye el vínculo de la sexualidad masculina y femenina y las respectivas identidades sexuales.

Esta construcción de las sexualidades masculina y femenina a partir del deseo humano es fundamental para entender la profunda transformación antropológica que tiene lugar en la actual sociedad de mercado, donde el individuo individualista (egoísta, narcisista y hedonista) deja de desear el deseo del "otro", no considera la subjetividad que supone el deseo del "otro" ni al otro en cuanto sujeto de su propia sexualidad, haciendo que su deseo sexual reduzca al "otro" a la condición de objeto del propio placer.

No es la diferencia de sexos o diferencias genitales (hoy quirúrgicamente alterables) lo que hace diferentes a un hombre y a una mujer, sino sus respectivas sexualidades construidas a partir de una cultura determinada y de sus particulares relaciones eróticas entre ellos; negar esta diferencia no es más que el atajo para negar el vínculo social que pueda existir entre el hombre y la mujer. Primero es en su familia y en la sociedad, donde niños, niñas y adolescentes son sujetos de una transmisión de lo que significa ser mujer y hombre, y después este "aprendizaje" se convierte en experiencia a partir de las relaciones entre ambos sexos.

Según esto, y en contra de toda apariencia, no son las diferencias entre los sexos las que fundan el vínculo entre ellos (aunque dicha diferencia sea condición necesaria para ello), sino al contrario, más bien el vínculo erótico, la relación entre ambos sexos, es la razón suficiente que funda y condiciona la alteridad y real diferencia entre los sexos. Prueba de ello, como se plantea más adelante, es cuando los vínculos sociales se quiebran, y el vínculo erótico se atrofia y elimina entre los sexos, que las identidades sexuales se confunden, se fragmentan, se pulverizan y colisionan entre ellas, al entrar en una suerte de big bang erótico característico de la sociedad moderna.

\section{Construcción social de las sexualidades}

Si la sexualidad y los usos del sexo son construcciones sociales, cada modelo de sociedad produce sus propias prácticas y relaciones sexua- 
les y formas de sexualidad. Así pues, los usos de la sexualidad y las relaciones sexuales o relaciones entre sexos responden siempre a un determinado modelo de organización social y más precisamente al modo como una sociedad instituye y norma su cohesión interna. Así mismo los actos sexuales significan los modos propios de socialidad en una determinada sociedad. Siempre que una sociedad se funda sobre una u otra forma de vínculo social y relaciones sociales, éstas no dejarán de expresarse en las prácticas y usos de la sexualidad; y de manera correspondiente las relaciones sexuales contribuirán a su manera a la reproducción de dichos vínculos o cohesión social ${ }^{8}$.

En las sociedades primitivas o comunales, organizadas y reguladas por el parentesco, por ejemplo, la sexualidad respondía tanto a una reproducción de la familia y la sociedad como a un reforzamiento de los vínculos del parentesco y de la comunidad. En la antigua Grecia la relación erótica se distinguía de la amistad o filial y del amor caritativo o ágape, como si todas estas formas de relación entre individuos pudieran complementarse con vínculos personales y en el reforzamiento de una ciudadanía (de la polis).

Otras sociedades contribuirían con nuevas y singulares formas de pensar y vivir la sexualidad. Así, para la antigua cultura hebrea la relación sexual entre el hombre y la mujer se designaba con el verbo conocer $(y d h)$, lo que podía significar tanto que en la relación sexual se expresaba y comunicaba lo más subjetivo e individual de la persona como que en dicha relación el reconocimiento comportaba una mutua identificación y vínculo entre el hombre y la mujer. "Adán conoció a Eva, su mujer, que concibió..." (Génesis, $4.1)^{9}$.

Pero no hay vínculo ni reconocimiento posibles sin la radical diferencia entre los sexos: "lo que el hombre y la mujer comparten no es una comunidad de intereses, de placeres, de pasiones, sino el gusto por su extrañeza recíproca, una mutua ignorancia insuperable" (Bruckner \& Finkielkraut, 1979:238). La tradicio-

Cfr. Michel Bozon, "Les significations sociales des actes sexuels", Actes de la Recherche en Sciences Sociales, n. 128, juin 1999.

9 Mientras que el conocer de la tradición griega comporta una posesión intelectual objetiva de la realidad ydh es más bien un conocimiento existencial entre sujetos, que comporta una particular relación personal entre ellos. 
nal cultura de las sociedades andinas ha hecho de la radical diferencia entre masculinidad y feminidad el símbolo de todas las diferencias y complementariedades en la naturaleza y en la sociedad, donde todo es qari y huarmi (hombre y mujer); una dualidad a la vez insuperable pero cuyos términos no existen el uno sin el otro, y cuya unión es por ello tan ilimitada como imposible, puesto que nunca logra la unidad entre ellos. Por eso en lengua kichwa la idea de matrimonio se expresa por una fórmula sintáctica: qari-huarmi.

Las tradicionales sociedades andinas son muy representativas de cómo la concepción del cuerpo humano y su participación en una misma corporalidad ("carne": aycha) social definen tanto las formas de pertenencia a una misma comunidad social como las diferencias, complementariedades y relaciones sexuales, e incluso regula las posibles violencias entre hombre y mujer ${ }^{10}$.
Para Hegel, quien funda la eticidad humana en el nivel más originario del vínculo y reconocimiento entre el hombre y la mujer, matriz y modelo de todos los otros vínculos y reconocimientos en la sociedad humana, la "lucha por el reconocimiento" (Kampf zur Erkennung) es siempre lucha por reconocer la igualdad en la diferencia; y en el caso de la sexualidad reconocer al "otro" en cuanto sujeto en el cuerpo objetivo del placer. En la construcción ética hegeliana el reconocimiento opera a tres niveles: funda el amor entre los sexos, la moral entre los individuos dentro de la sociedad civil y el derecho entre ciudadanos de la sociedad política ${ }^{11}$. Y donde no hay una tal "lucha por el reconocimiento" entre las personas, entonces se desatan en la sociedad humana todas las otras luchas, desde la lucha de clases hasta las lucha de sexos, para terminar en la "lucha de todos contra todos" (Hobbes).

10 Cfr. J. Sánchez Parga, ¿Por qué golpearla? 'Etica, estética y ritual en los Andes, CAAP, Quito, 1990; “Cuerpo y enfermedad en las representaciones indígenas de los Andes", en A.C. Defossez et al., Mujeres de los Andes. Condiciones de vida y salud, IFEA / Universidad Externado de Colombia, 1992.

11 Para Hegel estos tres niveles se articulan dialécticamente entre sí: el amor, la moral y el derecho se presuponen entre sí, al mismo tiempo que se completan mutuamente. Cfr. W. F. Hegel, Jenaer Realphilosophie von 1805/06, hrsg. Von J. Hoffmeister, Leipzig, 1931:206s. 


\section{Fracturas de la sexualidad y lucha de sexualidades en la sociedad de mercado}

No sólo la sociedad de mercado produce sus propios usos de la sexualidad, sus prácticas y relaciones sociales, de acuerdo a las fuerzas, lógicas e intereses del mercado, sino que también las formas de la sexualidad, sus usos y prácticas son producidos para el mercado y en función del mercado, adoptando la forma de marketing y mercancía, de oferta y demanda, de intercambios monetarios, de consumo. Siempre existió la venta del sexo mediante la prostitución, pero nunca alcanzó los niveles de negocio, de tráfico, de organización mafiosa y de explotación como en la sociedad actual.

Los nuevos significados sociales de la práctica sexual son obvios: cada grupo y cada individuo hacen de la sexualidad un uso propio y apropiado a las formas que adopta su individualismo: un uso egoísta, narcisista, hedonista, competitivo y consumidor. En este sentido se habla de "una nueva normatividad de la sexualidad en la época contemporánea" dictada desde los individuos ${ }^{12}$. Ahora bien, ¿cómo es posible establecer una normatividad cualquiera, sea esta sexual o de otro género, desde el individuo más individualista? Una normatividad, sea la que sea, sólo puede ser pensada y practicada desde un mínimo común, en la medida en que pueda ser mínimamente compartida, en cuanto regulación de una colectividad y en razón de un interés general que rebase los individuales ${ }^{13}$.

Lo que hoy está en cuestión no es tanto la sexualidad moderna en sí misma, sus usos y abusos, cuanto una nueva antropología, una "nueva economía psíquica del sujeto" (Melman), producto de la actual sociedad de mercado. En este sentido todas las posmodernas morfologías del sexo, toda la plural y diversa fenomenología de la sexualidad, tienen que ser entendidas y explicadas a partir de este "nuevo-ser-humano" con sus modos de existir individualistas y a partir de las fuerzas, lógicas e intereses del mercado capitalista, el

12 Cfr. Michel Bozon, La nueva normatividad de la sexualidad en la época contemporánea. Conferencia dictada el 26 de enero de 2009 en FLACSO, México.

13 Esta normatividad individual de la sexualidad corresponde a lo que Marie Astrid Dupret llama "desregulación y violencia" de las sexualidades posmodernas: Sexualidad posmoderna: de la desregulación a la violencia, en Ecuador Debate, n. 78. Diciembre, 2009. 
cual está obligado a "devastar" todos los usos, prácticas y relaciones sexuales pertenecientes al anterior modelo de sociedad ${ }^{14}$.

De la misma manera que la "destrucción productiva" del capital opera en todos los otros ámbitos e instituciones de la sociedad, igualmente el mercado capitalista necesita destruir todas aquellas prácticas y usos de la sexualidad que impiden su desarrollo, al mismo tiempo que produce nuevas relaciones sexuales y nuevos usos de la sexualidad, que promuevan sus ulteriores desarrollos y legitimen su consolidación. Más aún, puesto que la sexualidad siempre fue la matriz y modelo, símbolo, del vínculo social, el mercado capitalista necesita destruir, más que cualquier otra relación social, el carácter vinculante de la sexualidad y librar de tal vinculación a todos los usos de la sexualidad y liberar todas las sexualidades individuales, unas de las otras, para una competitividad ilimitada y encarnizada entre ellas.
No otra es en el fondo la "liberación sexual" de la posmodernidad: liberar la sexualidad de cualquier vínculo entre las personas, para desatar un "derecho al goce" sin responsabilidades entre ellas, y más bien investido de recíprocas violencias: "muy lejos de disminuir bajo el efecto de un progreso ineluctable dicha violencia (entre sexos) es actualmente más cotidiana, rabiosa y enloquecida en la misma medida que las mujeres se liberan"15.

En el "nuevo orden amoroso" (Bruckner \& Finkielkraut) de la posmodernidad no es el vínculo social entre los sexos sino el record de los orgasmos lo que persiguen todas las sexualidades, desencadenando nuevas $y$ violentas competitividades entre ellas. Son las vinculaciones de los individuos al mercado (la mercancía, la oferta y la demanda, el consumo) lo que fractura el vínculo en las relaciones personales, fetichizando el sexo y la sexualidad; de la misma manera que la fetichización

14 El concepto marxista de "devastación" y la "destrucción productiva" de Schumpeter definen la acción del capital, que destruye todo lo que en la sociedad impide su desarrollo, produciendo nuevas realidades sociales que lo consolidan: cfr. K.Marx, Das Kapital. Kritik der politischen Ökonomie, libro I, parte IV, cap. 13, 3 a) Dietz Verlag, Berlin, 1969; Joseph Schumpeter, Capitalisme, Socialisme et Démocratie, Payot, Paris, 1969.

15 "La emancipación femenina no liquida la agresión, le añade la odiosa dimensión del resentimiento. Atacar (hoy) a una mujer no es una actitud instintiva y salvaje del primate, es la reacción de un propietario ante la abolición de la esclavitud": Pascal Bruckner \& Alain Finkielkraut, El nuevo desorden amoroso, Ed. Anagrama, Barcelona, 1979:321 nota. 
de la mercancía por el capital comporta su creciente erotización y sexualización.

$\mathrm{Si}$ en la moderna sociedad de mercado domina una "economía libidinal" (Lyotard) es debido a los deslizamientos entre los ámbitos de la sexualidad y los de la mercancía: la intensidad libidinal de las relaciones entre sexos se ha ido desplazando progresivamente hacia la "intensidad libidinal del intercambio capitalista"16. Esto hace que "el deseo del capital" (Lyotard) resulte extraordinariamente difícil de reconocer, porque ha penetrado tanto todos los otros campos del deseo, incluido sobre todo el sexual, que llega a confundir unos deseos con otros. De ahí que donde aparece un supuesto intercambio erótico no haya más que un intercambio de intereses, de ofertas y demandas, de consumos...

\section{Bibliografía}

\section{BOZON, Michel}

1999 "Les significations sociales des actes sexuels", Actes de la Recherche en Sciences Sociales, n. 128 , juin.

2009 La nueva normatividad de la sexualidad en la época contem- poránea. Conferencia dictada el 26 de enero de 2009 en FLACSO, México.

BRUCKNER, P. \& FINKIELKRAUT, A. 1979 El nuevo desorden amoroso Barcelona: Ed. Anagrama.

DUPRET, Marie-Astrid

2009 "Sexualidad postmoderna: de la desregulación a las violencias", en Ecuador Debate, n. 78, diciembre.

DURKHEIM, Emile Durkheim

1968 Formes élémentaires de la vie religieuse, PUF, Paris.

HEGEL, W.F.

1931 Jenaer Realphilosophie von 1805/06, hrsg. Von J. Hoffmeister, Leipzig.

JEANVOINE, Michel Jeanvoine

2006 "Le corps et ses marques", Journal Francais de Psychiatrie, n. 24.

LE BRETON, David

1990 Anthropologie du corps et modernité, PUF, Paris.

LEENHARDT, Maurice

1971 Do Kamo. La personne et le mythe dans le monde mélanésien, Paris: Gallimard.

LERUDE, Martine

2003 "La feminidad: ¿cómo se construye?", en Ecuador Debate, n. 59, agosto.

16 Jean-Francois Lyotard, Economía libidinal, Fondo Cultura Económica, Buenos Aires, 1990. 
José Sánchez Parga

LYOTARD, Jean - Francois

1990 Economia Libidinal, Buenos Aires: Fondo de Cultura Económica.

MARX, K.

1969 Das Kapital. Kritik der politischen Ökonomie, Dietz Verlag, Berlin.

SANCHEZ PARGA, J.

1990 ¿Por qué golpearla? Etica, estética y ritual en los Andes, CAAP, Quito.
1992 "Cuerpo y enfermedad en las representaciones indígenas de los Andes", en A.C. Defossez et al., Mujeres de los Andes. Condiciones de vida y salud, IFEA / Universidad Externado, Colombia.

SCHUMPETER, J.

1969 Capitalisme, Socialisme et Démocratie, Paris: Payot. 